\title{
Differences in the Composition of Preterm and Term Human Milk during Early Lactation
}

\author{
JAMES A. LEMONS, ${ }^{(31)}$ LEMUEL MOYE, DANIEL HALL, AND MICHAEL SIMMONS
}

Department of Pediatrics, Indiana University School of Medicine and the James Whitcomb Riley Hospital for Children, Indianapolis, IN and the Department of Perinatology, Denver Children's Hospital, Denver, Colorado, USA

\begin{abstract}
Summary
The composition of human milk from mothers delivering prematurely (PT) and at term (T) was studied over the first weeks of lactation. Complete $24 \mathrm{~h}$ milk expressions were obtained by electric pump at weekly or biweekly intervals through 44 wk conceptual age (120 samples from 20 PT mothers and 28 samples from $7 \mathrm{~T}$ mothers). PT milk was found to contain significantly higher concentrations than $\mathbf{T}$ milk of the following nutrients: total nitrogen, protein nitrogen, sodium, chloride, magnesium and iron. No differences were found between $T$ and PT milk for nonprotein nitrogen, volume, solids, total calories, lactose, fat, fatty acids, potassium or calcium. The nutrients supplied to a 33 wk preterm infant fed 200 $\mathrm{ml} / \mathrm{kg} /$ day of "average" PT milk were in excess of theoretic intrauterine requirements for all substrates except calcium and phosphorus. PT human milk is theoretically more suitable for the premature infant than either mature or term human milk, but may be deficient in specific nutrients for the very low birth weight baby.
\end{abstract}

\section{Speculation}

The compositional differences in preterm milk (increased protein and mineral content) are generally characteristic of colostrum. These changes may therefore reflect a prolonged colostral phase in premature mothers who are establishing lactation by artificial means during periods of emotional stress. Thus, the changes denoted for preterm milk may reflect the circumstances surrounding the onset of lactation rather than the nutritional requirements of the preterm infant.

Controversy persists regarding the propriety of human milk for the feeding of preterm infants. Although insufficient data are available to determine exact nutritional requirements of the premature baby (16), Fomon et al. (10) have appropriately questioned the adequacy of mature breast milk in relation to estimated needs. While providing theoretically beneficial nonnutritional factors, mature human milk may be quantitatively deficient in protein, calcium, sodium and possibly other nutrients for the premature (10). Clinical studies of premature infants fed mature breast milk have yielded conflicting results $(8,14,19,25)$, although the data generally indicate that mature human milk does not provide optimal nutritional support to the low birth weight infant.

Recent investigations offer intriguing data which indicate that the composition of milk from mothers delivering prematurely differs from that of term mothers during the early weeks of lactation. Atkinson et al. (2) and Gross et al. (15) have confirmed a higher concentration of protein nitrogen in preterm milk, while Gross et al. also found an increased level of sodium in the premature mothers' milk. These findings suggest that preterm milk is theoretically more suitable for the premature infant than is mature milk. The present investigation was performed to assess, in a comprehensive fashion, the composition of milk from mothers delivering prematurely and at term over the same period of lactation. Further, the study was designed to control for many variables known to affect breast milk composition (stage of lactation, degree of breast emptying, diurnal variation and method of milk expression) $(9,17,21)$.

\section{MATERIALS AND METHODS}

Milk donors and sample collections. Complete $24 \mathrm{~h}$ expressions of milk from both breasts were obtained by a group of 20 preterm (PT) and 7 term (T) mothers at weekly or biweekly intervals following parturition. The mean gestational age of the preterm group was $33 \mathrm{wk}$ (range 27-37) and that of the term mothers 40 wk (range 39-4l). All pregnancies were considered normal without significant maternal disease. Each resulted in vaginal delivery of appropriately grown for gestational age infants. Prepregnancy weight and weight gain throughout pregnancy were within normal limits for each mother. The age, gravity and parity of the mothers in each group were similar. One set of twins was delivered in the preterm group.

Samples were collected on days 7, 14, 21 and 28 and then biweekly until a postconceptual age of $44 \mathrm{wk}$ was attained. All samples were collected into deionized plastic containers utilizing an electric breast pump (Egnell, Cary, IL). During the $24 \mathrm{~h}$ collection period, the samples were maintained at refrigerator temperature. The total volume of milk expressed was then measured, thoroughly mixed and aliquots obtained for substrate analysis. Each aliquot was maintained at $-80^{\circ} \mathrm{C}$ until future analysis.

Analytical methods. The following methods were employed in the analysis of the milk composition. Total caloric content was determined in triplicate for each sample, using the 1241 adiabatic bomb calorimeter (Parr Instrument Co., Moline, IL) following lyophilization of the milk sample. This method determines total caloric value of milk with a coefficient of variation of less than $0.5 \%$. Total solids were determined by lyophilization of a $10 \mathrm{ml}$ aliquot of milk. The difference between pre- and post-lyophilization weights of the milk yielded values for total water content as well as total solids. Total ash content was measured by placing an accurately weighed sample of milk in a $100^{\circ} \mathrm{C}$ oven for $24 \mathrm{~h}$, which was then transferred to a $540^{\circ} \mathrm{C}$ muffle furnace for $24 \mathrm{~h}$ and then reweighed. Osmolality was determined by modified freezing point depression (Advanced Instruments, Needham Heights, MA). Total nitrogen was determined by the KjeIdahl method (24). Nonprotein nitrogen and protein nitrogen were determined by the same method following acid precipitation of a milk sample with measurement of the nitrogen contents of the supernatant and precipitate respectively. Lactose content was determined by gas chromatography based upon a modification of the methodology of Brobst and Lott (4) and Brittain et al. (3). Total lipids were determined by the method of Frings and Dunn (12). The fatty acid composition of the total lipids was quantitated by gas chromatography utilizing the method of Nelson (23). Sodium and potassium were assayed by flame emission spectrophotometry, using Instrumentation Laboratories, Inc. (Lexington, MA) instrumentation with lithium as an internal standard. Chloride was determined by chloridometer, using amperometric end 
point detection. Calcium was measured by a modification of the method of Connerty and Briggs (7) and phosphorus by an automated colorimetric method (29). Iron and magnesium were quantitated by atomic absorption spectrophotometry (Instrumentation Laboratories, Lexington, MA, Model 353AAS).

All assays were performed in duplicate or triplicate and mean values for each sample utilized in final data analysis.

Statistical methods. Two categories of mothers were examined (PT and T). Since variables of interest (e.g., nitrogen concentration) can be influenced by the sample age and the volume of milk per sample, analysis of covariance methods were employed to adjust for the effects of these covariates. The covariance analysis was applied in two stages. First, a regression line relating the variable of interest and the covariates was computed for each mother. These regression lines were then pooled for all PT as well as for all $\mathrm{T}$ mothers (a procedure supported by the data). Comparisons between categories ( $T$ versus $\mathrm{PT}$ ) were then made by analyses of the pooled lines. Covariance analysis applied in this fashion corrects for the effects of the covariates (sample age and sample volume) both within and between categories, and appropriately weights the computed relationships by the number of sample points obtained from each mother.

\section{RESULTS}

The results of the analyses of both PT and T milk composition are presented in Table 1.

Volume: The volume of milk produced during a $24 \mathrm{~h}$ period exceeded $140 \mathrm{ml} / \mathrm{kg} /$ day for all samples in both the $\mathrm{T}$ and PT group. Mean $24 \mathrm{~h}$ volumes were similar in both groups. No significant effect of prematurity on the success of lactation was apparent.

Osmolality: The mean osmolality of the PT milk was 303.7 $\mathrm{mOsm} /$ liter, and that of T milk $302.1 \mathrm{mOsm} /$ liter. No significant change was detected in relation to duration of lactation or degree of prematurity.

Calories: Total caloric value was not different between the PT and $\mathrm{T}$ groups, with means of $73.16 \mathrm{kcal} / 100 \mathrm{ml}$ (or $21.95 \mathrm{kcal} / 30$ , $\mathrm{ml}$ ) and $72.27 \mathrm{kcal} / 100 \mathrm{ml}$ (or $21.68 \mathrm{kcal} / 30 \mathrm{ml}$ ) respectively. No change was noted in relation to the degree of prematurity or the duration of lactation. The mean caloric value of the milk samples, as determined by bomb calorimetric analysis, was $18.5 \%$ greater than that based upon calculation from protein, lactose and fat content (6).

Nitrogen: The concentration of total nitrogen (TN) and protein nitrogen (PN) were greater in the PT group than the $\mathrm{T}$ group during the first $4 \mathrm{wk}$ of lactation $(P<0.005)$ (Tables 1 and 2$)$. The TN was $\sim 11 \%$ greater in the PT group, while PN was $\sim 13 \%$ greater in the PT group. When compared with the "average" protein content of mature pooled breast milk of $0.88 \mathrm{~g} \%$ (18), the preterm infant fed the same volume of his own mother's milk would receive $\sim 67 \%$ more protein than when fed pooled donor milk during the first month of life. The concentrations of TN and PN decreased over the first 4 wk of lactation in both the T and PT groups. The rate of decline was the same for all PT and $\mathrm{T}$ mothers (Table 2). Similarly, TN and PN in both PT and T mothers demonstrated a significant $(P<0.005)$ inverse correlation with the volume of milk produced (Table 2); i.e., as the volume of sample increased, the asbsolute concentrations of TN and PN decreased. Nonprotein nitrogen (NPN) remained relatively constant during the period of study, comprising $\sim 13 \%$ of the TN or $\sim 33 \mathrm{mg}$ nitrogen $/ 100 \mathrm{ml}$.

Sodium and chloride: Both sodium and chloride were found in significantly greater concentrations in the PT group then the $\mathrm{T}$ group during the first $4 \mathrm{wk}$ of lactation $(P<0.001)$. Although the concentration declined for both sodium and chloride as the duration of lactation increased, levels similar to pooled mature milk were not reached until approximately 8 wk of lactation in the PT group. Based upon the "average" sodium cotnent of mature breast milk ( $7 \mathrm{mEq} /$ liter) (18), a preterm infant being fed his own mother's milk during the first month of life would receive $\sim 75 \%$ more sodium than when fed pooled mature milk. The rate of decline in sodium was the same for both $\mathrm{T}$ and $\mathrm{PT}$ mothers, with a significant $(P<0.005)$ inverse correlation with volume of milk produced (Table 2 ).

Potassium: Potassium concentrations were similar in both groups of mothers, and declined over the first $4 \mathrm{wk}$ of lactation.

Magnesium: Magnesium concentrations were minimally greater in the PT milk than the T milk $(P<0.005)$. The level did not appear to change during lactation, nor did the degree of prematurity have a detectable effect.

Iron: The concentration of iron in the PT milk was greater $(P$ $<0.005$ ) than that of the $\mathrm{T}$ milk throughout the first month of lactation ( 1.51 versus $0.84 \mathrm{mg} /$ liter, respectively). The level of iron was widely variable between women, without a detectable relationship to vitamin or iron supplementation. No significant change in concentration was noted over the first $8 \mathrm{wk}$ of lactation in the PT group.

Calcium, Phosphorus: The calcium concentration was the same in PT (289 mg/liter) and T (279 mg/liter) milk and did not change significantly over the first $8 \mathrm{wk}$ of lactation. The phosphorus content of PT milk (132.5 mg/liter) was significantly less $(P<$ $0.05)$ than that of $T$ milk $(152.2 \mathrm{mg} /$ liter $)$. The concentration did not change over the period of lactation studied. The calcium/ phosphorus ratio approached 2.0 in both groups (PT 2.2, T 1.8).

Solids: The amount of solids was similar in both groups (appropximately $12.8 \mathrm{~g} \%$ ). No effect of duration of lactation or degree of prematurity was evident.

Ash: Ash content was significantly greater $(P<0.005)$ in PT $(0.226 \mathrm{~g} \%)$ than $\mathrm{T}$ milk $(0.199 \mathrm{~g} \%)$. This may reflect the higher concentrations of sodium, chloride, magnesium and iron in PT milk.

Fat: No significant difference was found between the PT and T groups, nor was any effect of duration of lactation or prematurity evident. The mean concentration in the PT group was $3.28 \mathrm{~g} \%$, and in the T milk $3.02 \mathrm{~g} \%$.

Fatty Acids: The concentrations of 4 fatty acids, which together comprise $\sim 85 \%$ of the fatty acids found in human milk, are presented in Table 1 . No significant differences between the PT and $T$ group, or changes over the duration of lactation, are apparent.

Lactose: Lactose was not different between the PT and T groups (6.729 versus $6.761 \mathrm{~g} \%$, respectively). No significant effect of prematurity or duration of lactation was evident.

\section{DISCUSSION}

Results of the present investigation demonstrate significant differences in the composition of milk from mothers delivering prematurely versus mothers delivering at term, when sampled in the same manner over the same period of lactation. Total nitrogen, protein nitrogen, sodium, chloride, magnesium, and iron are present in PT milk in higher concentrations than in T milk. Phosphorus levels, on the other hand, are slightly lower in PT milk.

Other recent reports have documented a similar difference in composition of PT milk from $\mathrm{T}$ milk in relation to nitrogen content. Atkinson et al., (2) in analyzing serial $24 \mathrm{~h}$ milk collections from a group of preterm and term mothers, found a significantly higher total nitrogen and protein nitrogen content in PT milk which decreased during the first month of lactation. Schanler and Oh (26) demonstrated a higher nitrogen content in PT milk when compared with pooled donor milk. The findings of Gross et al. (15) were similar, although samples were collected only from a single feeding on each day of study.

However, significant discrepancies between the findings of the present study and other reports are evident in regard to several substrates. Anderson et al. (1) have recently reported a $20-30 \%$ higher energy content in PT milk during early lactation compared to T milk, due to a 20-30\% higher concentration of fat in PT milk. The fat concentration in the T milk increased from $1.85 \mathrm{~g} \%$ at 3 to 5 days lactation to $3.06 \mathrm{~g} \%$ at 15 to 18 days of lactation. In the PT milk the lipid concentration increased from an initial $3.0 \mathrm{~g} \%$ to $4.33 \mathrm{~g} \%$ by 15 to 18 days of lactation. As discussed by Anderson et al., complete emptying of the breast is imperative in order to 
Table 1 . The nutritional composition of PT and T milk during the early wk of lactation

\begin{tabular}{|c|c|c|c|c|c|c|c|c|c|c|}
\hline \multirow[b]{2}{*}{ Nutrient } & \multicolumn{2}{|c|}{$\begin{array}{l}\text { Sample } 1 \\
\text { (7 days) }\end{array}$} & \multicolumn{2}{|c|}{$\begin{array}{l}\text { Sample } 2 \\
\text { (14 days) }\end{array}$} & \multicolumn{2}{|c|}{$\begin{array}{l}\text { Sample } 3 \\
\text { (21 days) }\end{array}$} & \multicolumn{2}{|c|}{$\begin{array}{l}\text { Sample } 4 \\
\text { (28 Days) }\end{array}$} & \multirow{2}{*}{$\begin{array}{l}\begin{array}{l}\text { Sample } 6 \\
\text { (42 days) }\end{array} \\
\text { Preterm }\end{array}$} & \multirow{2}{*}{$\begin{array}{c}\begin{array}{c}\text { Sample } 8 \\
(\geq 56 \text { days })\end{array} \\
\text { Preterm }\end{array}$} \\
\hline & Preterm & Term & Preterm & Term & Preterm & Term & Preterm & Term & & \\
\hline $\begin{array}{l}\text { Calories } \\
(\text { Kcal/100 ml) }\end{array}$ & $\begin{array}{c}73.86 \pm 1.81^{3} \\
(20)\end{array}$ & $\begin{array}{l}73.62 \pm 3.32 \\
\text { (7) }\end{array}$ & $\begin{array}{l}74.59 \pm 1.96 \\
\text { (19) }\end{array}$ & $\begin{array}{c}71.81 \pm 3.57 \\
(7)\end{array}$ & $\begin{array}{c}73.84 \pm 1.89 \\
(17)\end{array}$ & $\begin{array}{c}70.40 \pm 3.28 \\
(7)\end{array}$ & $\begin{array}{c}73.33 \pm 2.14 \\
(17)\end{array}$ & $\begin{array}{l}72.71 \pm 1.88 \\
\text { (7) }\end{array}$ & $\begin{array}{c}70.10 \pm 1.424 \\
(14)\end{array}$ & $\begin{array}{c}76.33 \pm 2.34 \\
\text { (11) }\end{array}$ \\
\hline $\begin{array}{l}\text { Osmolality } \\
\text { (mOsm/liter) }\end{array}$ & $\begin{array}{l}302 \pm 2.89 \\
\quad(20)\end{array}$ & $\begin{array}{c}301.57 \pm 2.93 \\
(7)\end{array}$ & $\begin{array}{c}304.22 \pm 1.51 \\
(18)\end{array}$ & $\begin{array}{c}301.29 \pm 1.97 \\
(7)\end{array}$ & $\begin{array}{c}303.24 \pm 2.27 \\
(17)\end{array}$ & $\begin{array}{c}303.57 \pm 2.32 \\
(7)\end{array}$ & $\begin{array}{c}304.811 \pm 2.93 \\
(16)\end{array}$ & $\begin{array}{l}302 \pm 3.70 \\
\quad(7)\end{array}$ & $\begin{array}{c}303.15 \pm 2.96 \\
(13)\end{array}$ & $\begin{array}{c}304.80 \pm 2.09 \\
(10)\end{array}$ \\
\hline $\begin{array}{l}\text { Total nitrogen } \\
\text { (g/liter) }\end{array}$ & $\begin{array}{l}3.20 \pm 0.11 \\
(20)\end{array}$ & $\begin{array}{l}2.86 \pm 0.098 \\
\quad(7)\end{array}$ & $\begin{array}{l}2.68 \pm 0.12 \\
\quad(19)\end{array}$ & $\begin{array}{l}2.54 \pm 0.17 \\
\quad(7)\end{array}$ & $\begin{array}{l}2.61 \pm 0.11 \\
\quad(18)\end{array}$ & $\begin{array}{l}2.32 \pm 0.09 \\
\quad(7)\end{array}$ & $\begin{array}{l}2.38 \pm 0.11 \\
\quad(16)\end{array}$ & $\begin{array}{l}2.09 \pm 0.07 \\
\quad(7)\end{array}$ & $\begin{array}{l}2.39 \pm 0.09 \\
\quad(13)\end{array}$ & $\begin{array}{c}2.28 \pm 0.08 \\
(9)\end{array}$ \\
\hline $\begin{array}{l}\text { Nonprotein nitrogen } \\
\text { (g/liter) }\end{array}$ & $\begin{array}{c}0.31 \pm 0.03 \\
(8)\end{array}$ & $\begin{array}{l}0.32 \pm 0.03 \\
\quad(6)\end{array}$ & $\begin{array}{c}0.33 \pm 0.03 \\
\text { (9) }\end{array}$ & $\begin{array}{l}0.38 \pm 0.02 \\
\quad(4)\end{array}$ & $\begin{array}{c}0.37 \pm 0.04 \\
\quad(8)\end{array}$ & $\begin{array}{l}0.35 \pm 0.01 \\
\quad(5)\end{array}$ & $\begin{array}{c}0.32 \pm 0.02 \\
\quad(9)\end{array}$ & $\begin{array}{l}0.29 \pm 0.03 \\
\quad(4)\end{array}$ & $\begin{array}{l}0.35 \pm 0.05 \\
\text { (7) }\end{array}$ & $\begin{array}{c}0.32 \pm 0.02 \\
(6)\end{array}$ \\
\hline $\begin{array}{l}\text { Protein nitrogen }{ }^{1} \\
\text { (g/liter) }\end{array}$ & $\begin{array}{l}2.76 \pm 0.18 \\
\quad(8)\end{array}$ & $\begin{array}{l}2.55 \pm 0.13 \\
(6)\end{array}$ & $\begin{array}{c}2.39 \pm 0.16 \\
(9)\end{array}$ & $\begin{array}{l}1.97 \pm 0.14 \\
(4)\end{array}$ & $\begin{array}{c}2.17 \pm 0.14 \\
\quad(8)\end{array}$ & $\begin{array}{c}1.89 \pm 0.06 \\
\quad(5)\end{array}$ & $\begin{array}{c}1.90 \pm 0.09 \\
\quad(9)\end{array}$ & $\begin{array}{l}1.76 \pm 0.08 \\
\quad(4)\end{array}$ & $\begin{array}{l}1.99 \pm 0.10 \\
(7)\end{array}$ & $\begin{array}{l}1.96 \pm 0.10 \\
(6)\end{array}$ \\
\hline $\begin{array}{l}\text { Sodium }{ }^{1} \\
\quad(\mathrm{mEq} / \text { liter })\end{array}$ & $\begin{array}{l}17.23 \pm 1.88 \\
\quad(20)\end{array}$ & $\begin{array}{l}9.54 \pm 1.30 \\
(7)\end{array}$ & $\begin{array}{c}12.36 \pm 1.42 \\
(19)\end{array}$ & $\begin{array}{l}9.37 \pm 1.95 \\
\quad(7)\end{array}$ & $\begin{array}{l}10.51 \pm 0.96 \\
\quad(18)\end{array}$ & $\begin{array}{c}6.83 \pm 1.02 \\
(7)\end{array}$ & $\begin{array}{l}9.56 \pm 0.70 \\
(16)\end{array}$ & $\begin{array}{l}7.04 \pm 0.96 \\
(7)\end{array}$ & $\begin{array}{l}8.85 \pm 0.86 \\
\quad(13)\end{array}$ & $\begin{array}{c}7.14 \pm 0.51 \\
(9)\end{array}$ \\
\hline $\begin{array}{l}\text { Potassium } \\
\quad(\mathrm{mEq} / \text { liter })\end{array}$ & $\begin{array}{c}17.28 \pm 0.67 \\
(20)\end{array}$ & $\begin{array}{l}16.86 \pm 1.22 \\
\text { (7) }\end{array}$ & $\begin{array}{c}15.59 \pm 0.48 \\
\text { (19) }\end{array}$ & $\begin{array}{c}14.57 \pm 0.51 \\
(7)\end{array}$ & $\begin{array}{c}14.43 \pm 0.46 \\
\quad(18)\end{array}$ & $\begin{array}{l}13.94 \pm 0.55 \\
\text { (7) }\end{array}$ & $\begin{array}{c}13.75 \pm 0.39 \\
(16)\end{array}$ & $\begin{array}{c}13.00 \pm 0.51 \\
\text { (7) }\end{array}$ & $\begin{array}{c}14.43 \pm 0.81 \\
\quad(13)\end{array}$ & $\begin{array}{c}13.58 \pm 0.42 \\
(9)\end{array}$ \\
\hline $\begin{array}{l}\text { Chloride }^{1} \\
\text { (mEq/liter) }\end{array}$ & $\begin{array}{c}18.60 \pm 1.41 \\
\quad(20)\end{array}$ & $\begin{array}{c}13.90 \pm 1.57 \\
\text { (7) }\end{array}$ & $\begin{array}{l}14.35 \pm 1.23 \\
\quad(19)\end{array}$ & $\begin{array}{c}12.11 \pm 1.82 \\
(7)\end{array}$ & $\begin{array}{l}12.84 \pm 0.90 \\
\quad(18)\end{array}$ & $\begin{array}{c}10.53 \pm 1.17 \\
\text { (7) }\end{array}$ & $\begin{array}{c}12.65 \pm 0.68 \\
(16)\end{array}$ & $\begin{array}{c}10.46 \pm 0.91 \\
\text { (7) }\end{array}$ & $\begin{array}{c}11.98 \pm 0.61 \\
\quad(12)\end{array}$ & $\begin{array}{c}11.36 \pm 0.36 \\
\text { (9) }\end{array}$ \\
\hline $\begin{array}{l}\text { Iron }^{1} \\
(\mathrm{mg} / \text { liter })\end{array}$ & $\begin{array}{l}1.50 \pm 0.11 \\
(20)\end{array}$ & $\begin{array}{l}0.77 \pm 0.10 \\
(7)\end{array}$ & $\begin{array}{l}1.52 \pm 0.21 \\
\text { (19) }\end{array}$ & $\begin{array}{c}0.98 \pm 0.20 \\
(7)\end{array}$ & $\begin{array}{l}1.72 \pm 0.36 \\
(15)\end{array}$ & $\begin{array}{l}0.80 \pm 0.15 \\
(7)\end{array}$ & $\begin{array}{l}1.31 \pm 0.16 \\
(14)\end{array}$ & $\begin{array}{l}0.81 \pm 0.13 \\
(7)\end{array}$ & $\begin{array}{l}1.39 \pm 0.42 \\
\text { (11) }\end{array}$ & $\begin{array}{c}1.77 \pm 0.51 \\
(9)\end{array}$ \\
\hline $\begin{array}{l}\text { Calcium } \\
\text { (mg/liter) }\end{array}$ & $\begin{array}{c}293.11 \pm 16.35 \\
(20)\end{array}$ & $\begin{array}{c}293.14 \pm 7.61 \\
(7)\end{array}$ & $\begin{array}{c}266.32 \pm 15.03 \\
(19)\end{array}$ & $\begin{array}{c}274.44 \pm 12.76 \\
(7)\end{array}$ & $\begin{array}{c}281.74 \pm 13.36 \\
(18)\end{array}$ & $\begin{array}{c}279.51 \pm 9.50 \\
(7)\end{array}$ & $\begin{array}{c}282.45 \pm 11.73 \\
(16)\end{array}$ & $\begin{array}{c}267.46 \pm 12.58 \\
\text { (7) }\end{array}$ & $\begin{array}{c}309.79 \pm 16.02 \\
(13)\end{array}$ & $\begin{array}{c}314.4 \pm 11.63 \\
(9)\end{array}$ \\
\hline $\begin{array}{r}\text { Phosphorus } \\
\text { (mg/liter) }\end{array}$ & $\begin{array}{c}134.48 \pm 6.45 \\
(20)\end{array}$ & $\begin{array}{c}169.30 \pm 8.55 \\
(7)\end{array}$ & $\begin{array}{c}138.84 \pm 6.97 \\
(19)\end{array}$ & $\begin{array}{c}151.99 \pm 7.98 \\
(7)\end{array}$ & $\begin{array}{c}138.68 \pm 3.61 \\
(18)\end{array}$ & $\begin{array}{c}149.10 \pm 8.45 \\
(7)\end{array}$ & $\begin{array}{c}131.63 \pm 5.69 \\
(16)\end{array}$ & $\begin{array}{c}138.53 \pm 9.09 \\
\text { (7) }\end{array}$ & $\begin{array}{c}129.47 \pm 5.96 \\
(13)\end{array}$ & $\begin{array}{c}130.78 \pm 3.53 \\
(9)\end{array}$ \\
\hline $\begin{array}{l}\text { Solids } \\
\qquad(\mathrm{g} / 100 \mathrm{ml})\end{array}$ & $\begin{array}{c}12.52 \pm 0.29 \\
\quad(20)\end{array}$ & $\begin{array}{l}12.98 \pm 0.28 \\
\quad(7)\end{array}$ & $\begin{array}{l}12.85 \pm 0.22 \\
\quad(19)\end{array}$ & $\begin{array}{l}12.94 \pm 0.33 \\
(7)\end{array}$ & $\begin{array}{l}12.84 \pm 0.28 \\
\quad(18)\end{array}$ & $\begin{array}{c}12.64 \pm 0.29 \\
\text { (7) }\end{array}$ & $\begin{array}{l}12.50 \pm 0.26 \\
\quad(16)\end{array}$ & $\begin{array}{l}12.65 \pm 0.25 \\
\text { (7) }\end{array}$ & $\begin{array}{c}12.49 \pm 0.14 \\
(13)\end{array}$ & $\begin{array}{c}12.69 \pm 0.18 \\
\text { (9) }\end{array}$ \\
\hline $\begin{array}{l}\text { Ash } \\
\qquad(\mathrm{g} / 100 \mathrm{ml})\end{array}$ & $\begin{array}{l}0.25 \pm 0.01 \\
\quad(20)\end{array}$ & $\begin{array}{l}0.23 \pm 0.01 \\
\quad(7)\end{array}$ & $\begin{array}{l}0.22 \pm 0.01 \\
\quad(19)\end{array}$ & $\begin{array}{l}0.21 \pm 0.01 \\
\quad(7)\end{array}$ & $\begin{array}{l}0.22 \pm 0.01 \\
\quad(15)\end{array}$ & $\begin{array}{l}0.19 \pm 0.01 \\
\quad(6)\end{array}$ & $\begin{array}{l}0.21 \pm 0.01 \\
\quad(14)\end{array}$ & $\begin{array}{l}0.18 \pm 0.01 \\
\quad(6)\end{array}$ & $\begin{array}{l}0.21 \pm 0.01 \\
\quad(11)\end{array}$ & $\begin{array}{c}0.21 \pm 0.01 \\
\quad(9)\end{array}$ \\
\hline $\begin{array}{l}\text { Fat } \\
\qquad(\mathrm{g} / 100 \mathrm{ml})\end{array}$ & $\begin{array}{l}3.10 \pm 0.17 \\
(19)\end{array}$ & $\begin{array}{l}2.98 \pm 0.28 \\
\quad(7)\end{array}$ & $\begin{array}{l}3.42 \pm 0.18 \\
\quad(18)\end{array}$ & $\begin{array}{l}3.00 \pm 0.26 \\
(7)\end{array}$ & $\begin{array}{l}3.53 \pm 0.22 \\
\quad(17)\end{array}$ & $\begin{array}{l}3.04 \pm 0.33 \\
\quad(7)\end{array}$ & $\begin{array}{l}3.24 \pm 0.16 \\
(16)\end{array}$ & $\begin{array}{l}3.07 \pm 0.21 \\
\quad(7)\end{array}$ & $\begin{array}{l}3.43 \pm 0.16 \\
\quad(11)\end{array}$ & $\begin{array}{l}3.46 \pm 0.32 \\
\quad(9)\end{array}$ \\
\hline $\begin{array}{l}\text { Palmitic acid } \\
\text { (\% of total fat) }\end{array}$ & $\begin{array}{c}25.11 \pm 0.47 \\
(7)\end{array}$ & $\begin{array}{l}23.17 \pm 0.36 \\
\text { (4) }\end{array}$ & $\begin{array}{c}23.87 \pm 0.79 \\
(7)\end{array}$ & $\begin{array}{c}23.87 \pm 0.79 \\
(6)\end{array}$ & $\begin{array}{c}23.00 \pm 1.15 \\
(8)\end{array}$ & $\begin{array}{c}25.10 \pm 2.02 \\
(6)\end{array}$ & $\begin{array}{c}22.18 \pm 1.38 \\
(6)\end{array}$ & $\begin{array}{l}23.20 \pm 1.23 \\
\text { (6) }\end{array}$ & $\begin{array}{c}24.17 \pm 2.27 \\
\text { (3) }\end{array}$ & $\begin{array}{c}21.74 \pm 1.87 \\
(5)\end{array}$ \\
\hline $\begin{array}{l}\text { Stearic acid } \\
\quad(\% \text { of total fat })\end{array}$ & $\begin{array}{c}9.19 \pm 0.54 \\
(7)\end{array}$ & $\begin{array}{l}8.45 \pm 0.51 \\
\quad(4)\end{array}$ & $\begin{array}{c}9.10 \pm 0.70 \\
(7)\end{array}$ & $\begin{array}{l}8.82 \pm 0.72 \\
\quad(6)\end{array}$ & $\begin{array}{c}8.90 \pm 1.29 \\
(8)\end{array}$ & $\begin{array}{l}9.42 \pm 0.43 \\
\quad(6)\end{array}$ & $\begin{array}{c}10.12 \pm 1.17 \\
(6)\end{array}$ & $\begin{array}{l}9.47 \pm 0.99 \\
\quad(6)\end{array}$ & $\begin{array}{c}9.07 \pm 0.56 \\
(3)\end{array}$ & $\begin{array}{c}8.28 \pm 0.61 \\
(5)\end{array}$ \\
\hline $\begin{array}{l}\text { Oleic acid } \\
\text { (\% of total fat) }\end{array}$ & $\begin{array}{c}36.80 \pm 0.80 \\
\text { (7) }\end{array}$ & $\begin{array}{l}36.80 \pm 1.58 \\
\quad \text { (4) }\end{array}$ & $\begin{array}{c}34.67 \pm 0.60 \\
\text { (7) }\end{array}$ & $\begin{array}{l}34.35 \pm 4.32 \\
(6)\end{array}$ & $\begin{array}{c}38.46 \pm 2.02 \\
(8)\end{array}$ & $\begin{array}{l}36.03 \pm 1.30 \\
(6)\end{array}$ & $\begin{array}{c}35.35 \pm 0.96 \\
(6)\end{array}$ & $\begin{array}{l}38.77 \pm 1.62 \\
\text { (6) }\end{array}$ & $\begin{array}{c}37.27 \pm 1.68 \\
\text { (3) }\end{array}$ & $\begin{array}{c}36.34 \pm 3.91 \\
(5)\end{array}$ \\
\hline $\begin{array}{l}\text { Linoleic acid } \\
\text { (\% of total fat) }\end{array}$ & $\begin{array}{c}12.21 \pm 1.29 \\
(7)\end{array}$ & $\begin{array}{c}14.02 \pm 1.29 \\
\text { (4) }\end{array}$ & $\begin{array}{c}12.29 \pm 2.18 \\
(7)\end{array}$ & $\begin{array}{l}13.33 \pm 0.62 \\
(6)\end{array}$ & $\begin{array}{c}13.99 \pm 2.20 \\
(8)\end{array}$ & $\begin{array}{l}12.97 \pm 2.20 \\
(6)\end{array}$ & $\begin{array}{l}12.97 \pm 2.05 \\
\text { (11) }\end{array}$ & $\begin{array}{l}13.05 \pm 1.67 \\
\text { (6) }\end{array}$ & $\begin{array}{c}12.63 \pm 3.00 \\
\text { (3) }\end{array}$ & $\begin{array}{c}20.74 \pm 8.01 \\
(5)\end{array}$ \\
\hline $\begin{array}{l}\text { Volume } \\
\quad \text { (per } 24 \mathrm{hr} \text { sample) }\end{array}$ & $\begin{array}{c}392.91 \pm 76.58 \\
(20)\end{array}$ & $\begin{array}{c}530.71 \pm 78.82 \\
\text { (7) }\end{array}$ & $\begin{array}{c}457.11 \pm 91.83 \\
(19)\end{array}$ & $\begin{array}{c}603.14 \pm 72.29 \\
(7)\end{array}$ & $\begin{array}{l}504.17 \pm 102.29 \\
(18)\end{array}$ & $\begin{array}{c}601.72 \pm 60 \\
\text { (7) }\end{array}$ & $\begin{array}{c}492.12 \pm 53.19 \\
(17)\end{array}$ & $\begin{array}{l}599.57 \pm 68.47 \\
\text { (7) }\end{array}$ & $\begin{array}{c}595.79 \pm 111.26 \\
\text { (14) }\end{array}$ & $\begin{array}{c}676.27 \pm 132.47 \\
\text { (11) }\end{array}$ \\
\hline
\end{tabular}


Table 2. The effects of volume and sample age on various substrate concentrations is presented, as well as the statistical difference in these substrate values between $T$ and PT milk

\begin{tabular}{|c|c|c|c|c|c|}
\hline Variable & $\begin{array}{l}\text { Slope pooled regression } \\
\text { coefficient for } \\
\text { sample age }\end{array}$ & $\begin{array}{c}\text { Slope pooled regression } \\
\text { coefficient for } \\
\text { volume }\end{array}$ & $\begin{array}{l}\text { Preterm adjusted } \\
\text { mean }^{1}\end{array}$ & $\begin{array}{c}\text { Term adjusted } \\
\text { mean }^{1}\end{array}$ & $P$ value \\
\hline Total nitrogen & $-0.0946(P<0.005)$ & $-0.00043(P<0.005)$ & $266 \mathrm{mg} / 100 \mathrm{ml}$ & $238 \mathrm{mg} / 100 \mathrm{ml}$ & $P<0.005$ \\
\hline Nonprotein nitrogen & $-0.0015(\mathrm{NS})$ & $0.000026(\mathrm{NS})$ & $33 \mathrm{mg} / 100 \mathrm{ml}$ & $34 \mathrm{mg} / 100 \mathrm{ml}$ & NS \\
\hline Protein nitrogen & $-0.0865(P<0.005)$ & $-0.000463(P<0.005)$ & $225 \mathrm{mg} / 100 \mathrm{ml}$ & $193 \mathrm{mg} / 100 \mathrm{ml}$ & $P<0.005$ \\
\hline Sodium & $-0.9023(P<0.005)$ & $-0.00529(P<0.005)$ & $12.12 \mathrm{mEq} /$ liter & $7.62 \mathrm{mEq} /$ liter & $P<0.001$ \\
\hline
\end{tabular}

${ }^{1}$ The mean concentration of substrate over the period of study after correcting for the effects of volume and sample age.

obtain a representative milk sample for fat analysis. Hytten (17) has demonstrated that the fat content of human milk increases from 1 to approximately $6 \mathrm{~g} \%$ during the progressive emptying of a breast. Should only a portion of the breast be emptied, one may expect a significantly lower fat content and decreased total caloric value, as well as dilutional alterations in the concentration of water soluble substances. In contrast to the data of Anderson et $a l$., results of the present study indicate that the total fat and energy contents of both PT and T milk are similar.

Furthermore, the caloric values of the PT and T milk are somewhat higher than previously reported for human milk (when based upon bomb calorimetric measurements). Interestingly, a difference between the calculated and measured caloric contents of human milk was also apparent. In the present investigation, total caloric value of breast milk as determined by bomb calorimetry exceeded calculated energy content (based upon protein, lactose and fat concentration) (6) by $\sim 18 \%$. The reasons for this discrepancy are unclear, but may reflect either the inclusion of all combustible substrate in the bomb calorimetry method, or cumulative (but systematic) error in the measurement of individual substrates. Further investigation is necessary to substantiate this observation.

Another discrepant finding reported by Gross et al. (15) was that of a lower concentration of lactose during the first month of lactation in PT milk than in the T milk. Although of interest, this finding could not be verified in the present investigation. The higher sodium content of PT milk observed in the present study was also found by Gross et al.

The physiologic basis for the differences in substrate concentrations between PT and T human milk remains unclear. Some of the differences (e.g., the increased protein content) may be of potential benefit to the very low birth weight infant during the early wk of life. The increased iron content of the preterm milk is of considerable interest. The levels demonstrated in the present study are 2- to 3-fold greater than previously reported (11). The significance and rationale for this finding are unknown at the present time. It is our impression that these differences are real and do not reflect alterations due to method of collection, success of lactation, or volume of milk produced. However, the changes noted in the preterm milk are generally characteristic of colostrum, with an increased protein and mineral content. It may be that a premature mother who is establishing lactation by artificial means during a period of emotional stress may undergo a prolonged colostral phase. Thus, the changes denoted for PT milk may, in fact, reflect the traumatic circumstances surrounding the onset of lactation, rather than the needs of the baby.

The practical significance of these compositional differences to the infant is unknown at this time, although one may speculate upon the nutritional adequacy of the PT milk based upon estimated requirements of individual nutrients for the preterm infant. Table 3 denotes the calculated increments of various nutrients in the fetus during normal intrauterine growth from 33 through 40 wk gestation (based upon the work of Ziegler et al. (30)). It must be emphasized that intrauterine growth rate is an arbitrary standard for postnatal growth of infants. In fact, such a rate of growth is rarely achieved postnatally. Further, at this point, there is no evidence that failure to achieve such growth rates is in any way harmful to the low birth weight infant; on the contrary, overzeal-
Table 3. Calculated intrauterine increments of various nutrients (per $k g$ per day) from 33-40 wk gestation ${ }^{1}$

\begin{tabular}{lcccccc}
\hline & \multicolumn{7}{c}{ Gestational age (wk) } \\
\cline { 2 - 7 } & 33 & 34 & 35 & 36 & 38 & 40 \\
\hline Weight (g) & 14.1 & 14.1 & 13.4 & 13.3 & 7.5 & 5.0 \\
Protein (g) & 1.84 & 1.87 & 1.81 & 1.80 & 1.06 & 0.73 \\
$\mathrm{Na}(\mathrm{mEq})$ & 1.0 & 1.0 & 0.94 & 0.93 & 0.49 & 0.29 \\
$\mathrm{~K}(\mathrm{mEq})$ & 0.6 & 0.57 & 0.56 & 0.54 & 0.31 & 0.17 \\
$\mathrm{Cl}(\mathrm{mEq})$ & 0.7 & 0.7 & 0.60 & 0.54 & 0.22 & 0.09 \\
$\mathrm{Mg}(\mathrm{mq})$ & 2.49 & 2.59 & 2.56 & 2.68 & 1.88 & 1.45 \\
$\mathrm{Ca}(\mathrm{mg})$ & 100.3 & 107.3 & 110.3 & 117.1 & 100.2 & 87.8 \\
$\mathrm{P}(\mathrm{mg})$ & 63.9 & 67.8 & 69.2 & 73.5 & 60.7 & 52.0 \\
Fat (g) & 1.82 & 1.88 & 1.88 & 1.98 & 1.66 & 1.45 \\
\hline
\end{tabular}

' Adapted from Ziegler, et al. (21).

ous efforts to sustain rapid growth rates may carry significant risks (13). With this caution in mind, one may compare the daily intrauterine increment for a variety of substrates with the actual intake which would be provided a 33 wk preterm infant fed his mother's breast milk (Table 4). Requirements postnatally will exceed intrauterine increments and will reflect the relative efficiency of absorption and retention of the premature infant. Such requirements have been estimated by the Committee on Nutrition of the American Academy of Pediatrics (5) for most nutrients. Based upon these estimates, an infant fed $170-200 \mathrm{ml} / \mathrm{kg} /$ day of his own mother's milk will receive an excess of the majority of substrates required to sustain intrauterine growth rate. Further, the volumes of milk produced by the preterm mother indicate that lactation can be established successfully utilizing a mechanical pump following premature delivery.

Although adequate for most nutrients, human milk (preterm or term) appears to contain insufficient amounts of calcium, phosphorus and possibly iron to meet the requirements of the low birth weight infant. Delayed bone mineralization $(22,27,28)$ and rickets (20) have been documented in premature infants being fed breast milk or cow's milk formulas containing varying amounts of calcium and vitamin $D$. Although the etiology of defective bone mineralization may be complex and involve hormonal and/or substrate imbalance, most investigators feel that dietary substrate deficiency may be the single most important factor. Ziegler et al. (30) and Shaw et al. (27) have demonstrated that intrauterine accretion rates of calcium exceed $100 \mathrm{mg} / \mathrm{kg} /$ day, and of phosphorus $50 \mathrm{mg} / \mathrm{kg} /$ day, during the last $8 \mathrm{wk}$ of pregnancy. Although absorption of calcium by preterm infants is relatively efficient from breast milk (65\%), net uptake is only $20 \%$ of estimated intrauterine requirements. The amount of calcium and phosphorus in PT milk is similar to previously reported values for malture milk (9). It may be anticipated, therefore, that bone mineralization may be deficient in premature infants fed their own mother's milk during early infancy.

In summary, results of the present investigation indicate that a preterm infant's own mother's breast milk is theoretically a more appropriate source of nutrient than either term milk or pooled mature breast milk. However, some nutrients (e.g., calcium, phosphorus) are apparently present in an insufficient amount to support normal growth. Appropriate supplementation of the milk 
Table 4. Nutrient intake of infant fed the "average" PT milk at $200 \mathrm{ml} / \mathrm{kg} /$ day

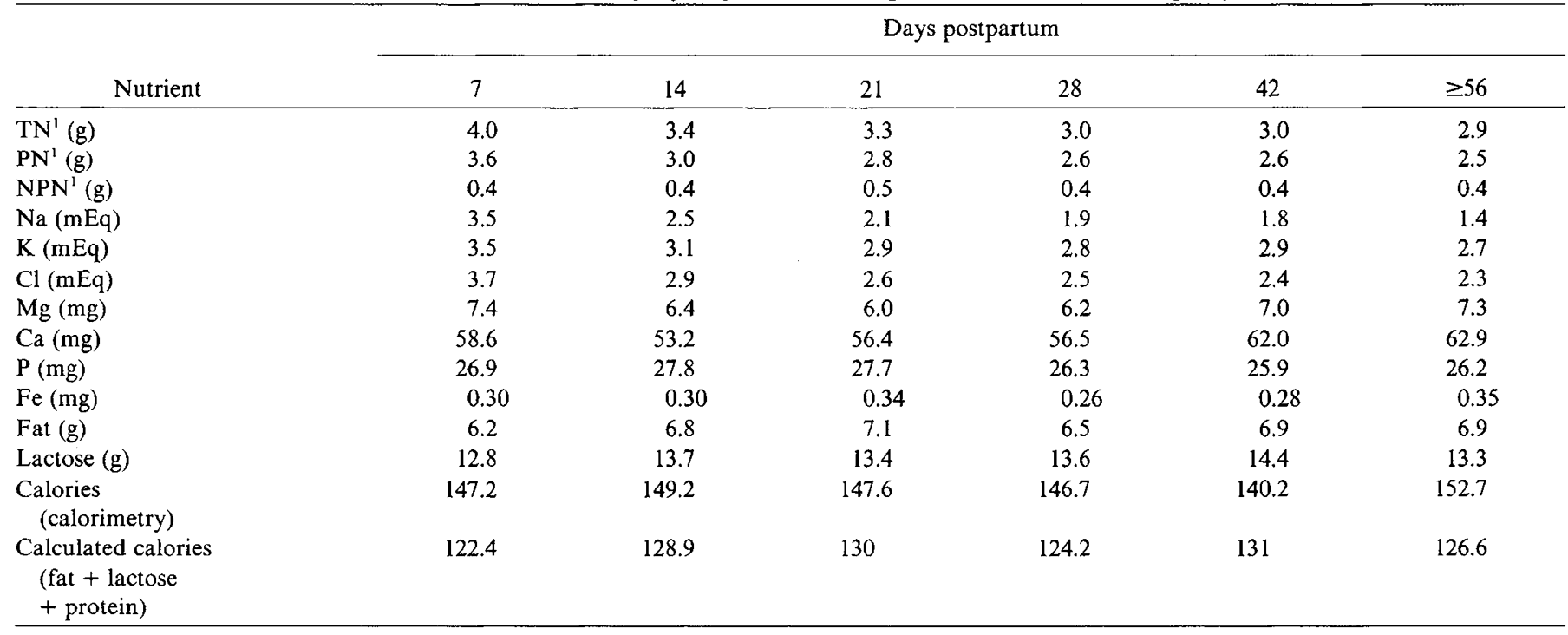

${ }^{1}$ Equivalent of $\mathrm{g}$ protein, obtained by multiplying $\mathrm{g}$ nitrogen $\times 6.38$.

may be warranted for these and possibly other factors. Controlled clinical trials are necessary to document the propriety of preterm human milk for the premature infant, and particularly for the very low birth weight baby.

\section{REFERENCES AND NOTES}

1. Anderson, G. H., Atkinson, S. A., and Bryan, M. H.: Energy and macronutrient content of human milk during early lactation from mothers giving birth prematurely and at term. Am. J. Clin. Nutr., 34: 258 (1981)

2. Atkinson, S. A., Bryan, M. H., and Anderson, G. H.: Human milk: difference in nitrogen concentration in milk from mothers of term and premature infants. $J$. Pediatr., 93: 67 (1978)

3. Brittain, G. D., Sullivan, J. E., and Schewe, L. R.: Silylation in the presence of $\mathrm{H}_{2} \mathrm{O}$ : The development of a commercial reagent for silylating aqueous solutions of hydroxy and polyhydroxy compound. In: Domsky, I. I. and Perry, J. A.: Recent Advances in Gas Chromatography p 223 (Dekker Company, New York, NY, 1971).

4. Brobst, K. M., and Lott, C. E.: Determination of some components in corn syrup by gas-liquid chromatography of the trimethylsilyl derivatives. J. Cereal Chem., 43: 35 (1966).

5. Committee on Nutrition of the American Academy of Pediatrics: Nutritional needs of low-birth-weight infants. Pediatrics, 60: 519 (1977)

6. Composition of Feeds, Agriculture Handbook, No 8-1, Washington DC, 1977, United States Department of Agriculture.

7. Connerty, H. V., and Briggs, A. R.: Determination of serum calcium by means of sodium alizarin sulfonate. Clin. Chem., 11:716 (1965).

8. Davies, D. P.: Adequacy of expressed breast milk for early growth of preterm infants. Arch. Dis. Child., 52: 296 (1977)

9. Fomon, S. J., and Filer, L. J., Jr.: Milks and formulas. In: Fomon, S. J.: Infant Nutrition p. 359 (W. B. Saunders Company, Philadelphia, PA, 1974).

10. Fomon, S. J., Ziegler, E. E., and Vazques, H. D.: Human milk and the small premature infant. Am. J. Dis. Child., 131: 463 (1977).

11. Franson, G-B., and Lönnerda'l, B.: Iron in human milk. J. Pediatr., 96: 380 (1980).

12. Frings, C. S., and Dunn, R. T.: A colorimetric method for determination of total serum lipids based on the sulfo-phospho-vanillin reaction. Am. J. Clin. Path., 53: 89 (1970).

13. Goldman, H. I., Goldman, J. S., Kaufman, I., and Liebman, O. B.: Late effects of early dietary protein intake on low birth weight infants. J. Pediatr., 85: 764 (1974).

14. Gordon, H. H., Levine, S. Z., and McNamara, H.: Feeding of premature infants: a comparison of human and cow's milks. Am. J. Dis. Child., 73: 442 (1947).
15. Gross, S. J., David, R. J., Bauman, L., and Tomarelli, R. M.: Nutritional composition of milk produced by mothers delivering preterm. J. Pediatr., 96: 64l (1980).

16. Heird, W. C., and Anderson, T. L.: Nutritional requirements and methods of feeding low birth weight infants. Curr. Probl. Pediatr., 7: 3 (1977).

17. Hytten, F. E.: Clinical and chemical studies in human lactation. Brit. Med. J., 1 : 175 (1954).

18. Jenness, R.: The composition of human milk. Sem. Perinatal. 3: 225 (1979).

19. Kagan, B. M., Hess, J. H., Lundeen, E., Shafer, K., Parker, J. B., and Stigall, C.: Feeding premature infants: a comparison of various milks. Pediatrics, 15: 373 (1955).

20. Kalkarni, P. B., Hall, R. T., Rhodes, P. G., Sheehan, M. B., Collenbach, M. D., German, D. R., and Abramson, S. J.: Rickets in very low-birth-weight infants. J. Pediatr., 96: 249 (1980).

21. Lawrence, R. A.: Biochemistry of human milk. In: Breast Feeding: a Guide for the Medical Profession p. 44. (The C. V. Mosby Company, St. Louis, 1980).

22. Minton, S. D., Steichen, J. J., and Tsang, R. C.: Bone mineral content in term and preterm appropriate-for-gestational-age infants. J. Pediatr., 95: 1037 (1979).

23. Nelson, G. J.: Extraction of plasma and serum. In: Nelson, G. J.: Blood Lipids and Lipoproteins: Quantitation, Composition and Metabolism p. 10 (John Wiley and Sons Inc., New York, 1972).

24. Official Methods of Analysis of the Association of Official Analytical Chemists, edition 11, Washington, 1979, Association of Official Analytical Chemists.

25. Raiha, N. C. R., Heinonen, K., Rassin, D. K., and Gaull, G. E.: Milk protein quantity and quality in low-birthweight infants: I. Metbolic responses and effects on growth. Pediatrics, 57: 659 (1976).

26. Schanler, R. J., and Oh, W.: Composition of breast milk obtained from mothers of premature infants as compared to breast milk obtained from donors. J. Pediatr., 96: 679 (1980).

27. Shaw, J. C. L.: Evidence for defective skeletal mineralization in low-birthweight infants: The absorption of calcium and fat. Pediatrics, 57: 16 (1976).

28. Steichen, J. J., Gratton, T. L., Tsang, R. C.: Osteopenia of prematurity: The cause and possible treatment. J. Pediatr., 96: 528 (1980).

29. Technicon Instrument Company, Tarrytown, New York.

30. Ziegler, E. E., O'Donnell, A. M., Nelson, S. E., and Fomon, S. F.: Body composition of the reference fetus. Growth, 40: 329 (1976).

31. Requests for reprints should be addressed to: Dr. J. A. Lemons, Department of Pediatrics, Indiana University School of Medicine, 1100 West Michigan Street, Indianapolis, Indiana 46223.

32. This research was supported in part by Ross Laboratories, 625 Cleveland Avenue, Columbus, $\mathrm{OH}$ and the National Institutes of Health, Bethesda, MD, Grant PHS 401 HD 12467-01.

33. Received for publication March 4, 1981.

34. Accepted for publication July 1, 1981. 